\section{Diagnostik und Therapie von Kopfschmerzen um die Jahrtausendwende}

Anlässlich der Jahrestagung der Schweizerischen Kopfwehgesellschaft in Aarau im Oktober 1999 haben wir versucht, für den Praktiker die wichtigsten Aspekte unseres Verständnisses von Kopfweh darzustellen. Die Artikel dieser Sondernummer beinhalten weitgehend die Beiträge aus dem Praktikernachmittag. Für das Verständnis von Kopfweh scheinen einige Dinge von herausragender Bedeutung, weshalb ich sie hier hervorheben möchte. Da ist auf der einen Seite der sogenannte Primäre Kopfschmerz, dessen Ursache viel zu sehr Gegenstand wissenschaftlicher Auseinandersetzung geworden ist. Hansruedi Isler (s.u.) zeigt wesentliche Grundbedingungen für die Entstehung dieses Kopfwehtyps. Dieses Kopfweh tritt meist vorübergehend auf ohne objektive Evidenz eines gewebeschädigenden Prozesses [1]. Das Gehirn besitzt ein schmerzmodulierendes System, das durch zahlreiche sehr individuelle Mechanismen beeinflusst werden kann und das offensichtlich auch verschiedenste Triggermechanismen kennt. Getreu dieser Erkenntnis versuchen die Autoren, nicht nur dieses Wissen zu ver- mitteln, sondern auch auf die seltenen gefährlichen Kopfwehursachen einzugehen und konkrete Therapieoptionen vorzustellen. Dies gilt besonders für spezielle Kopfwehprobleme bei Frauen und Kindern.

Bei dieser Betrachtungsweise von Kopfweh dürfen wir Sonderformen primärer Kopfschmerzen nicht vergessen, bei denen Beziehungen zwischen trigeminalem und autonomem System (Hypothalamus) existieren ("cluster headache", SUNCT-Syndrom und paroxysmale Hemikranie). Auch die Erkenntnisse der Neurogenetiker werden unser Verständnis mancher Kopfweh bald verändern. Ich denke an die hemiplegische Migräne, das Migräne-Koma und die episodische Ataxie Typ 2, die kausal KalziumKanal-Mutationen (CACNA1A) zugeordnet werden [2].

Die Gefahr, den Wald vor lauter Bäumen nicht zu sehen, ist bei einem derart häufigen Problem, wie dem Kopfweh sicher nicht von der Hand zu weisen. Deshalb wollen wir auf diesem Wege dazu beitragen, das Wissen über Kopfweh und die Zusammenarbeit zwischen Praktikern und Spezialisten zu fördern.

Prof. Dr. med. U. W. Buettner, Aarau

1 Fields HL. Central pain modulation in headache. Cephalalgia 1999;19:296.

2 Tournier-Lasserve E. CACNA1A mutations:

hemiplegic migraine, episodic ataxia type 2 , and the others. Neurology 1999;53:3-4. 